\title{
Inhomogeneous chemical evolution of r-process elements
}

B. Wehmeyer', M. Pignatari, and F.-K. Thielemann

Citation: AIP Conference Proceedings 1743, 040009 (2016); doi: 10.1063/1.4953301

View online: http://dx.doi.org/10.1063/1.4953301

View Table of Contents: http://aip.scitation.org/toc/apc/1743/1

Published by the American Institute of Physics 


\title{
Inhomogeneous Chemical Evolution of r-process Elements
}

\author{
B. Wehmeyer ${ }^{1, a), b)}$, M. Pignatari ${ }^{1,2}$ and F.-K. Thielemann ${ }^{1}$ \\ ${ }^{1}$ Univ Basel, Dept. Phys., Klingelbergstr. 82, CH-4056 Basel, Switzerland \\ ${ }^{2}$ Konkoly Observatory, Research Centre for Astronomy and Earth Sciences, Hungarian Academy of Sciences, \\ Konkoly Thege Miklós út 15-17, H-1121 Budapest, Hungary \\ ${ }^{a)}$ Speaker \\ b) Corresponding author: benjamin.wehmeyer@unibas.ch
}

\begin{abstract}
We report the results of a galactic chemical evolution (GCE) study for r-process- and alpha elements. For this work, we used the inhomogeneous GCE model "ICE", which allows to keep track of the galactic abundances of elements produced by different astrophysical sites. The main input parameters for this study were: a) The Neutron Star Merger (NSM) coalescence time scale, the probability of NSMs, and for the sub-class of "magneto-rotationally driven Supernovae" ("Jet-SNe"), their occurence rate in comparison to "standard" Supernovae (SNe).
\end{abstract}

\section{Introduction}

The "rapid neutron-capture process" (r-process) is responsible for about half of the heavy elements in our Galaxy (e.g., [4]). Although the process seems to be evident since many years, its true astrophysical site(s) are not fully identified, yet. GCE is a powerful tool to examine the history of element production in our Galaxy. We use the inhomogeneous GCE code "ICE" to investigate the evolution of several alpha- and an r-process element.

\section{Method}

Our GCE model "ICE" has numerous features, which details can be found in [6]. For convenience, we summarize the main parameters in the following. We simulate a volume of $(2 \mathrm{kpc})^{3}$, cut into sub-cubes of $(50 \mathrm{pc})^{3}$ each. The total simulation time is $13.6 E 9$ years, with time steps of $1 \mathrm{My}$ each. Every step, there is primordial matter infall into the simulation volume, governed by an initially rising and eventually exponentially decreasing rate over time:

$$
\dot{\mathrm{M}}(t)=a \cdot t^{b} \cdot e^{-t / \tau} .
$$

Then, the total mass in the sub-cubes is calculated. From this, the star formation rate per volume and time step is calculated. Star formation occurs under the assumption of a Schmidt law (power 1,5) and a Salpeter IMF (with slope $-2,35$ ). The probability of a star formation event is scaled with density, so star formation occurs preferably in a region swept up by a previous SN event. Newly born stars inherit the element abundances out of which they were formed. The life time of a newly born star is calculated via the Geneva Stellar Evolution and Nucleosynthesis Group formula

$$
\log (\mathrm{T})=(3.79+0.24 \cdot \mathrm{Z})-(3.10+0.35 \cdot \mathrm{Z}) \log (\mathrm{M})+(0.74+0.11 \cdot \mathrm{Z}) \log ^{2}(\mathrm{M}),
$$

where $\mathrm{T}$ is the lifetime in units of $10^{6} \mathrm{yrs}, \mathrm{Z}$ the metallicity, and $\mathrm{M}$ the mass in solar masses. Stars with $\mathrm{M}<8 \mathrm{M}_{\odot}$ end their life in a white dwarf and pollute their environment only locally. Stars $M>8 \mathrm{M}_{\odot}$ end their life in a core-collapse Supernova (CCSN) with an energy of the order of 1 Bethe, polluting a surrounding ISM of the mass of $5 \cdot 10^{4} \mathrm{M}_{\odot}$. The parameters $P_{S N I a}, P_{N S M}$, and $P_{J e t}$ determine, which fraction of newly born stars detonate in a Supernova type Ia, NSM, or Jet-SN event, instead. If a star/double star system detonates in such a way, the element enrichment of the surrounding ISM is considered to be different. SNIa highly enrich their surrounding in iron (Fe). NSMs are evident sites for an r-process and enrich their surrounding in r-process elements. However, they have an additional delay 


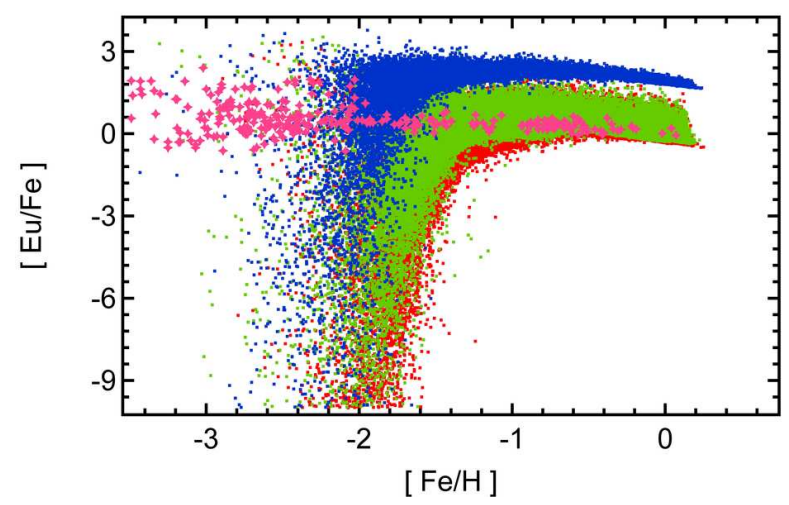

FIGURE 1. Influence of coalescence time scale and NSM-probability on Eu-Abundances in GCE. Magenta stars represent observations. Red dots correspond to model star abundances as in Argast et al. (2004). Green dots illustrate the effect on the abundances if the coalescence time scale of NSM is shorter. Blue dots show the abundance change if the probability of HMS binaries to later merge in a NSM is increased. Figure taken from [6].

time $t_{\text {coal }}$ between the CCSN of the progenitor stars and the inward spiral movement until the actual unification of both neutron stars is achieved. Jet-SNe (e.g., [7]) also produce r-process elements, but explode directly from their progenitor stars and are therefore able to enrich the ISM at significantly lower metallicities then NSM are able to.

\section{Results}

We examine the effects of the variation of the parameters $t_{c o a l}, P_{N S M}$, and $P_{J e t}$ on GCE.

\section{Coalescence time-scale and NSM probability}

The variation of coalescence time-scale and NSM probability have the following effects on GCE:

1. Lower coalescence time-scale results in earlier enrichment of europium and vice versa. While the two neutron stars coalesce, the surrounding ISM has time to evolve (to increase its metallicity). Therefore, the less time passes between the CCSN and the NSM, the lower the metallicity where the enrichment in r-process elements occurs. If the coalescence time-scale is longer, the ISM has more time to evolve, and therefore the enrichment in r-process elements happens later (at higher metallicities).

2. An increased probability of NSMs shifts the enrichment curve towards higher abundances and slightly towards lower metallicities, and vice versa. With higher NSM probability, the rate of NSM per time step is higher, and therefore (at a constant CCSN rate), more r-process elements are ejected. This shifts the abundance ratio towards higher r-process abundances. Consequently - also at lower metallicities - the production rate is higher compared to the standard case. This also shifts the abundance curve slightly to the left.

3. No combination of coalescence time-scale and NSM probability is able to reproduce the observed galactic r-process abundances.

\section{Probability of Jet-SNe}

Since Jet-SNe explode directly from a progenitor, their contribution to GCE differs from that of NSMs (who have additional coalescence time between progenitor CCSN and the actual NSM event).

1. Higher probability of Jet-SNe leads to a higher abundance in r-process elements and vice versa. The higher the rate of Jet-SNe per time-step, the higher the abundance of r-process in the ISM.

2. Jet-SNe are able to provide early abundance of r-process elements. This is due to the fact that they explode directly from a progenitor and do not have to wait for a coalescence time-scale (like NSMs). In the coalescence time, the surrounding ISM has time to evolve (and therefore increase its metallicity). Jet-SNe do not have an 


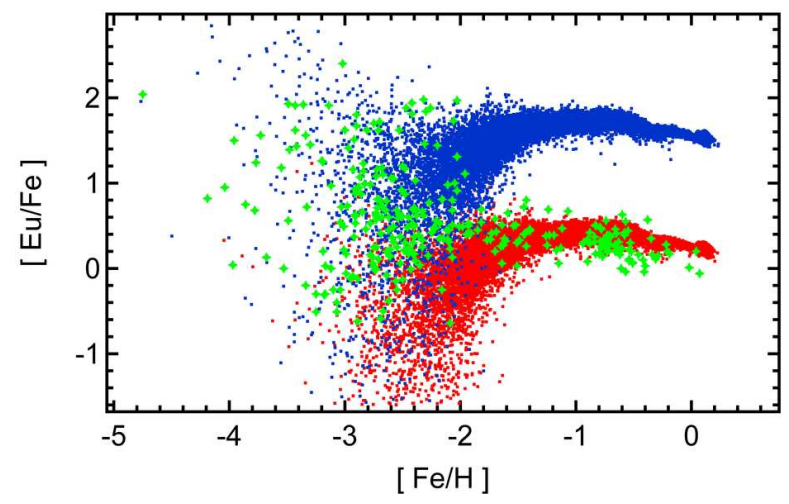

FIGURE 2. Influence of increased Jet-SN probabilities on Eu-Abundances in GCE. Green stars represent observations. Red dots represent model star abundances based on [7], the Jet-SN probability has been chosen to follow the observations at [Fe/H] $>-1.5$. A good value seems to be 1 per mill of high mass stars to end up in a Jet-SN. Blue dots illustrate the effect on the abundances if the Jet-SN probability is increased to one per cent. Figure taken from [6].

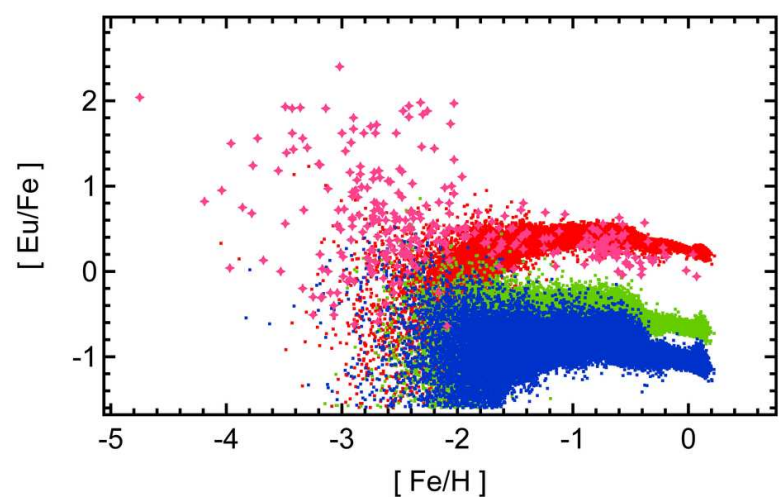

FIGURE 3. Same as Figure 2, but with decreased probabilities. Green and blue dots represent a Jet-SN probability of 0.1 per mill and 0.01 per mill, respectively. Figure taken from [6].

coalescence time-scale and therefore the surrounding ISM metallicity is lower when the Jet-SN event is taking place, permitting r-process enrichment at low metallicities.

3. Jet-SNe permit a large spectrum in r-process element abundances. Jet-SNe are able to produce r-process elements at low metallicities. At that stage of the evolution of the Galaxy, the ISM is highly inhomogeneous. This permits the existence of regions where many Jet-SNe have occured, as well as regions where only few Jet-SNe have occured. This, together with the fact that Jet-SNe are very rare events (of the order of up to $1 \%$ of all CC$\mathrm{SNe}$, estimated from pulsar/magnetar surveys, e.g., [1]) leads to a large variations in the levels of abundances in early stars. As a consequence, we can observe a large spread in r-process abundances at low metallicities.

\section{Combination of sites}

When both sites (NSMs and Jet-SNe) are employed in GCE, their contributions overlap. Therefore, good combinations for the parameters $t_{\text {coal }}, P_{N S M}$, and $P_{J e t}$ have to be found in order to match the observed stellar abundances. Although Jet-SNe might explain the observed abundances at lower metallicities, NSM start to contribute at later stages of the simulation. We use the above parameter explorations to tune the contributions of both astrophysical r-process production sites and find the following parameter set to match the observed abundances. $P_{\mathrm{NSM}}=0.021, P_{\mathrm{Jet}-\mathrm{SN}}=0.1$ per mill, $t_{\text {coal }}=10 \mathrm{My}$. The results for the model with these parameters in comparison with observations are shown in Figure 4. 


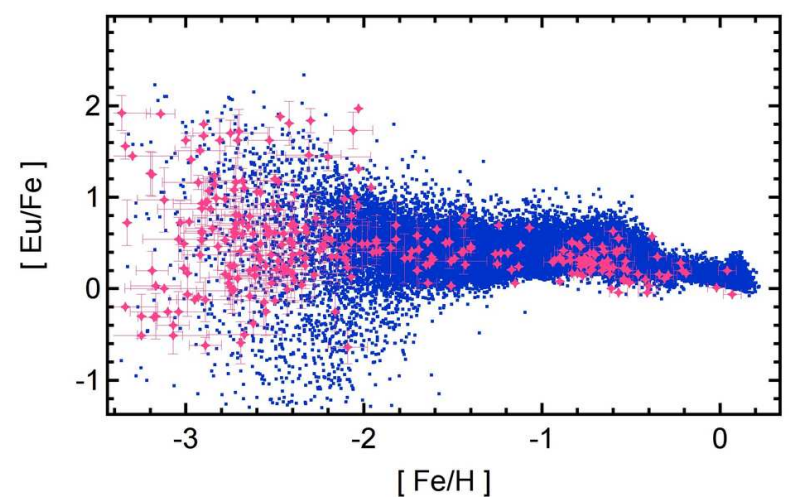

FIGURE 4. Evolution of Eu-abundances in GCE including both Jet-SN and NSM as r-process sites. Magenta stars represent observations (with observational errors; however, magenta stars at low metallicities which carry only horizontal errors represent upper limits), whereas blue dots represent model stars. Model parameters are $P_{\mathrm{NSM}}=0.021, P_{\mathrm{Jet}-\mathrm{SN}}=0.1$ per mill, $t_{\mathrm{coal}}=$ 10My. Figure taken from [6].

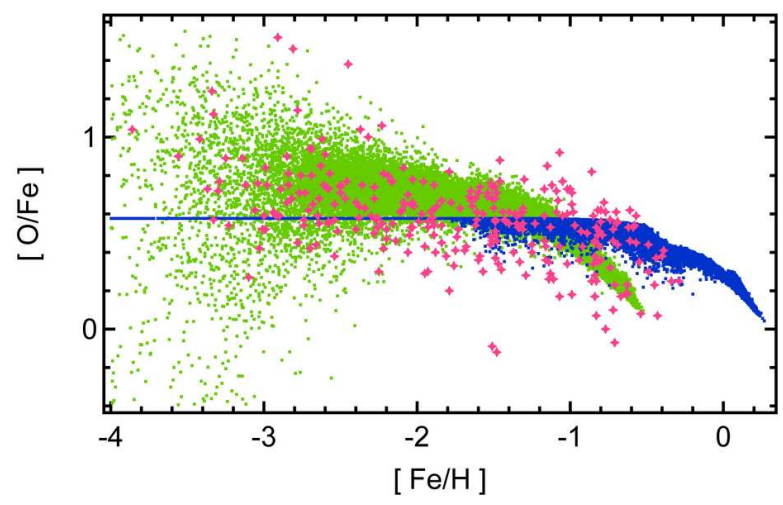

FIGURE 5. Same GCE-model as in Fig. 4; Green dots show the abundance evolution of Oxygen when canonical Nomoto et al. (1997) / Thielemann et al. (1996) yields are employed, while blue dots eventually extent to a single value at low metallicities if $a d$ hoc yields are applied. (Cf. Section b)) for further discussion. Figure taken from [6].

\section{Yields from core-collapse Supernovae}

While inhomogeneous GCE models might explain the abundances of r-process elements correctly, there is the question whether they might overpredict the abundance spread of the alpha-elements. This problem might originate in the problem that GCE models still rely on nucleosynthesis yields coming from still artificially simulated and calculated $\mathrm{SN}$ events. Considering that explosion energies might increase with the compactness of the stellar core (i.e. progenitor mass), the heavier alpha-elements and iron might be enhanced as a function of progenitor mass. On the other hand $\mathrm{O}, \mathrm{Ne}$, and $\mathrm{Mg}$ yields are dominated by hydrostatic burning and also increase with progenitor mass (e.g., [3]). This could permit to obtain more constant alpha/Fe ratios over a wide mass range, although the total amount of ejecta differs (increases) as a function of progenitor mass. To trace this problem, we ran a simulation where we replace the canonical Nomoto et al. (1997) / Thielemann et al. (1996) SN-Yields by ad hoc yields (who still fit the observed yields of SN1987A), which in principle represent a $[\mathrm{O} / \mathrm{Fe}]=0.5$ production ratio over all metallicities and progenitors. The outcome of this simulation run is, that the abundance spread of $[\mathrm{O} / \mathrm{Fe}]$ can be totally eliminated (until approximately $[\mathrm{Fe} / \mathrm{H}]=-1.8$, where $\mathrm{SNe}$ type Ia start to contribute). In Figure 5 we show the abundance evolution versus metallicity of our standard model in comparison to a model where ad hoc yields are employed. We might summarize that present stellar yields are responsible of the predicted [alpha/Fe] spread, independently from the $[\mathrm{r} / \mathrm{Fe}]$ spread. Therefore, more realistic CCSN yields from progenitor-dependent, self-consistently modelled explosions are highly needed for GCE simulations. 


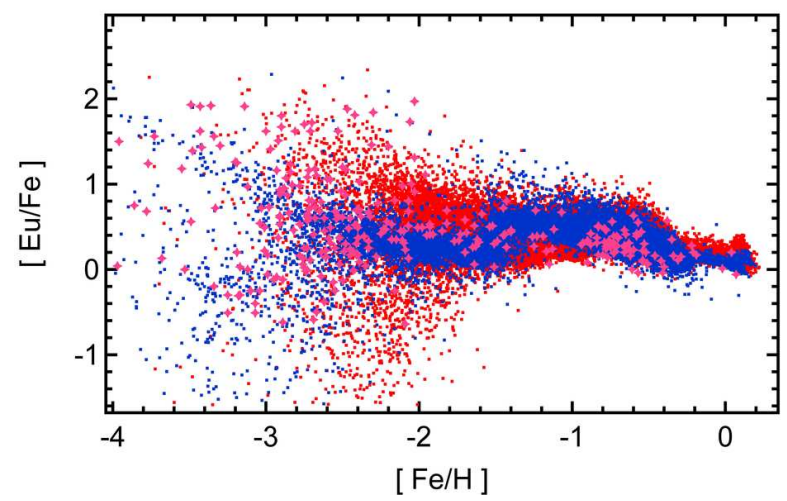

FIGURE 6. Effect of slightly increased sweep-up mass on GCE. Magenta stars represent observations. Red dots show model stars as per our reference model. Blue dots represent a model where every CCSN pollutes $2 \cdot 10^{5} \mathrm{M}_{\odot}$ of ISM. The dominant effect of this increased sweep up mass is to decrease the scatter of abundances and to shift the abundance curve slightly towards lower metallicities. Figure taken from [6].

\section{Swept-up mass}

As described in section b), every CCSN explodes with a blast energy of the order of 1 Bethe. This leads to a contamination of the order of $5 \cdot 10^{4} \mathrm{M}_{\odot}$ of ISM. Recent sophisticated GCE models run by, e.g., [5], and [2], use an alternative (SPH) blast method with pollution mass of the order of $3 \cdot 10^{6} \mathrm{M}_{\odot}$, and $(2-8) \cdot 10^{5} \mathrm{M}_{\odot}$, respectively. Such mixing with the ISM would require explosion energies of the order of $10-100$ Bethe, which is not expected for typical SNe. In a model run, we examine the outcome of a variation of the explosion energy of a CCSN (and therefore a changing swept-up mass). The outcome is, that we - in principle - can reproduce the main characteristics of their results. Therefore, the explosion energies of CCSNe have a high impact on GCE, but also the possible effect of other mixing processes which will have to be analyzed. Therefore, more realistic progenitor- and metallicity dependent explosion energies of CCSNe are highly needed in GCE simulations, as well as better knowledge of mixing processes.

\section{Conclusions}

1. NSM alone have difficulties to explain abundances at low metallicities. An earlier site cures this (e.g., Jet-SNe).

2. The spread in r-process elements at low metallicities can be explained by inhomogenieties.

3. Yields from SNe still have to be improved. They have large impact on GCE.

4. Explosion energies for whole spectrum of progenitors are highly needed for GCE.

\section{ACKNOWLEDGMENTS}

MP thanks the support from the Swiss National Science Foundation (SNF) and the "Lendület-2014" Programme of the Hungarian Academy of Sciences (Hungary). BW and FKT are supported by the European Research Council (FP7) under ERC Advanced Grant Agreement No. 321263 - FISH, and the Swiss National Science Foundation (SNF). The Basel group is a member in the COST Action New CompStar.

We thank the CETUP* 2015 organizers for a successful conference and local financial support.

\section{REFERENCES}

[1] Kramer M., 2009, in Strassmeier K. G., Kosovichev A. G., Beckman J. E., eds, Proc. IAU Symp. 259, Cosmic Magnetic Fields: From Planets, to Stars and Galaxies. Cambridge Univ. Press, Cambridge, p. 485

[2] Shen, S., Cooke, R., Ramirez-Ruiz, E., Madau, P., Mayer, L., Guedes, J., 2015 ApJ, 807, 115

[3] Thielemann F.-K., Nomoto K., Hashimoto M., 1996, ApJ, 460, 408

[4] Thielemann F.-K. et al., 2011, Progress Part. Nucl. Phys., 66, 346 
[5] van de Voort, F., Quataert, E., Hopkins, P. F., Keres, D., Faucher-Giguère, C.-A., 2015 MNRAS 447 140-148, DOI:10.1093/mnras/stu2404

[6] Wehmeyer, B., Pignatari, M., Thielemann, F.-K., 2015 MNRAS 452 (2), 1970-1981

[7] Winteler, C., Käppeli, R., Perego, A., Arcones, A., Vasset, N., Nishimura, N., Liebendörfer, M., Thielemann, F.-K., 2012 ApJ 750 L22 